\title{
Evaluation of in vitro Dissolution Profile and Physicochemical Characterization of Polymer Based Formulations of Sparingly Soluble Rosuvastatin
}

\author{
K. M. Yasif Kayes Sikdar ${ }^{1}$, Md. Shahoriar Nazir ${ }^{2}$, Md. Mahbubul Alam ${ }^{1}$, \\ Md. Raihan Sarkar ${ }^{1}$ and Sad Al Rezwan Rahman ${ }^{3}$ \\ ${ }^{1}$ Department of Pharmaceutical Technology, Faculty of Pharmacy, University of Dhaka, \\ Dhaka-1000, Bangladesh \\ ${ }^{2}$ Department of Pharmacy, Faculty of Pharmacy, University of Dhaka, Dhaka-1000, Bangladesh \\ ${ }^{3}$ Bangladesh Reference Institute for Chemical Measurement (BRiCM), Dhaka-1205, Bangladesh
}

(Received: November 3, 2021; Accepted: December 12, 2021; Published (web): December 26, 2021)

\begin{abstract}
Rosuvastatin (RVT) is a BCS class II antilipidemic crystalline drug, which exhibits low bioavailability due to its very poor aqueous solubility; therefore, it is challenging to develop a proper formulation of RVT. To enhance solubility and bioavailability of this API, an attempt has been made by implementing solid dispersion technique. Solid dispersion (SD) technique is a solubility enhancing technique where one or more active entities are dispersed in an inert medium (matrix or carrier) at solid state. In this study, different ratios of Kollicoat ${ }^{\circ}$ IR (KIR) and Kollidon ${ }^{\circledR}$ 90F (KF90) polymers were used with API to prepare various formulations by physical mixing (PM) and SD approaches; here solvent evaporation technique was used whereas methanol was used as solvent which was completely evaporated from the homogenously dispersed system by placing in a water-bath at $60-65^{\circ} \mathrm{C}$ and then in oven for 30 minutes at $50{ }^{\circ} \mathrm{C}$. Among the formulations, RVT-KF90 SD formulations showed the most promising result in in-vitro study in terms of drug release profile (78.04-99.21\%) in comparison to pure RVT (63.1\%) and physical mixing of RVT with those polymers. USP dissolution apparatus type II was used at $37^{\circ} \mathrm{C} \pm 0.5^{\circ} \mathrm{C}$ with 50 rpm to conduct the in-vitro experiment. The experiment also unraveled that the dissolution of RVT improved with increasing the amounts of polymers. Subsequently, stability of the developed formulations was conducted by Fourier transforms infrared spectroscopy (FTIR) and differential scanning calorimetry (DSC) as well as scanning electron microscopy (SEM). The results obtained from FTIR ensured no involvement of any significant drug-excipient interaction. Moreover, the DSC study signified thermal stability at high temperature. Besides, the SEM micrograph illustrated homogenous distribution of RVT in the polymer and transformation of crystal-like RVT into amorphous formulations.
\end{abstract}

Key words: Rosuvastatin, low solubility, physical mixing, solid dispersion, solvent evaporation.

\section{INTRODUCTION}

Formulating suitable dosage form is highly challenging for drugs which possess low aqueous solubility. ${ }^{1}$ The drug's water solubility has a significant impact on its oral dissolution rate. Orally administered medicines' dissolving rate, absorption, and bioavailability are all limited by their water solubility. ${ }^{1,2}$ Biopharmaceutical classification system

Correspondence to: Md. Raihan Sarkar

Cell: +8801705948057;

Email: raihan.rezvi@du.ac.bd

Dhaka Univ. J. Pharm. Sci. 20(2): 199-211, 2021 (December) DOI: https://doi.org/10.3329/dujps.v20i2.57170
(BCS) categorizes drug substrates into four categories, poorly water soluble drugs fall into the second and fourth category. ${ }^{2,3}$ The Noyes-Whiteny equation describes that dissolution rate of a API is directly proportionate to its solubility properties. ${ }^{4}$ Among the other factors to improve drug release, solubility is the most important factor to be concerned of. ${ }^{5-7}$ Several attempts have been made to upsurge the solubility of poorly soluble APIs from BCS class II group such as- salt formation, nanotechnology, co-crystallization, co-solvency, chemical modification, $\mathrm{pH}$ adjustment, hydrotropic, 
solubilizing agents, particle size reduction, physical modification, nanosuspension micronization, alteration of the crystal habit, self-microemulsifying drug delivery system, usage of complexing agents, by different surfactants, microemulsions, dispersion in carriers e.g. solid solution, solid dispersion. ${ }^{3,8}$

Solid dispersion (SD) system refers to the dispersed system where one or more active entities are dispersed in an inert medium (carrier or matrix) at solid state. ${ }^{9,10}$ Sekiguchi and Obi in their experiment used hot-melt method to prepare their system and urea as carrier excipient. ${ }^{9}$ Although later on hot-melt method became less popular due to some limitations such as active component and carrier must be miscible in molten form, active ingredient and carrier must be also thermo stable in high temperature etc. ${ }^{9}$ That's why solvent evaporation method became more popular. ${ }^{7}$ Fusion method, milling/co-grinding, spraydrying, supercritical fluid processing, and so on are also some approaches to prepare solid dispersion system in small or large scale. ${ }^{13} \mathrm{SD}$ system has an advantage over the conventional tablet/capsule as it reduces the crystal properties of drug substrates which cause immediate dissolution of the SD formulated tablet in the gastrointestinal fluid. ${ }^{11,12}$

Rosuvastatin Calcium (RVT) was approved by the US drug regulation authority, USFDA in 2003 as the seventh member of the statin family. ${ }^{14,15}$ In early stage of cholesterol biosynthesis, 3-hydroxy-3 methylglutaryl CoA (HMG-CoA) gets converted into mevalonate with the help of HMG-CoA reductase. ${ }^{14-}$

${ }^{16}$ It is hypothesized that the statin family members reduce the cholesterol level by inhibiting HMG-CoA reductase. ${ }^{14-16}$ RVT is a fully synthetic statin compound which was discovered by screening a series of pyrimidine-substituted dihydroxy-6heptenoates consisting of a sulphonyl functional group incorporated to decline lipophilicity and thus increase selectivity for the liver. ${ }^{15}$

RVT is a sparingly soluble crystalline BCS class II drug which is widely used for its antilipidemic activity. ${ }^{14,17,18}$ Due to low solubility in water $(0.33$ $\mathrm{mg} / \mathrm{ml}$ ), this antilipidemic drug demonstrates poor bioavailability $(20 \%)$ when it is taken orally. ${ }^{19}$
To improve the oral bioavailability of RVT hitherto several strives have been made. Among them selfnanoemulsifying drug delivery system $^{20-23}$, microparticle based drug delivery system $^{24-27}$, liquisolid technology ${ }^{28,29}$, solid dispersion technique ${ }^{30}$ are some notable approaches.

Kollicoat ${ }^{\circledR}$ IR (KIR) is a graft copolymer of polyethyleleglycol (PEG)-polyvinlylpyrrollidon (PVP) which was introduced by BASF Chemical Co. (Ludwigshafen, Germany) to design an immediate release dosage form. ${ }^{31}$ KIR is recommended for preparing SD system because of its interesting nonionic hydrophilic characteristics as well as along with gastrointestinal tract, its solubility does not change..$^{32-34}$ Moreover, it has surface-active characteristics and former studies showed that KIR is effective for SD system preparation which improves bioavailability of some BCS Class II APIs. ${ }^{32-36}$ Kollidon ${ }^{\circledR}$ 90F (KF90) is a commercial grade of polyvinylpyrroydone polymer series which is usually used as a binder. ${ }^{19,37}$ Potentiality of the aforementioned polymers were first time explored with RVT to enhance dissolution rate preparing $\mathrm{SD}$ formulations, since the available data is not enough to justify the polymer combinations required to enhance the dissolution rate of highly valuable drug molecule RVT.

The present study was focused on the enhancement of in vitro dissolution rate of RVT by a solid dispersion (SD) system, here solvent evaporation technique was used. The formulations were prepared with two different polymers as carrier, KIR and KF90. The system was further assessed by Fourier transform infrared (FTIR) spectroscopy for compatibility study, differential scanning calorimetry (DSC) for thermal stability checking, and scanning electron microscopy (SEM) for checking the surface morphology of the formulation. Moreover, in vitro dissolution studies of SD formulations were compared with the physical mixtures (PMs) which had the same ratio of active and polymers as in SDs as well as with the pure drug. 


\section{MATERIALS AND METHODS}

Materials. Rosuvastatin calcium (RVT), Kollicoat ${ }^{\circledR}$ IR (KIR) and Kollidon ${ }^{\circledR}$ 90F (KF90) were purchased from BASF, Germany. Sodium dihydrogen phosphate, sodium phosphate dibasic dihydrate, methanol, sodium hydroxide were bought from Merck, Germany. Other chemical agents and distilled water were collected and prepared from the Biopharmaceutics laboratory, Department of Pharmaceutical Technology, University of Dhaka, Bangladesh.

\section{Methods}

Preparation of physical mixing formulations (PMs). Pure active RVT and polymers (KIR and KF90) were weighed accurately by four-digit digital balance (Mettler Toledo, Switzerland). The measured RVT was mixed and triturated separately with KIR and KF90 in a properly cleaned mortar at different ratios of 1:1,1:2,1:3, and 1:4 to ensure homogenous mixture. Then the mixtures were sieved (36-micron test sieve, $300 \times 75 \mathrm{~mm}$ ) to get the uniformity of the particles in the mixtures. Finally, the mixing formulations were named as F1, F2, F3, F4, F5, F6,
F7 and F8 and kept in a desiccator at ambient temperature before further analysis (Table 1).

Preparation of SD formulations by solvent evaporation technique. Methanol was used as solvent to prepare solid dispersion system in this method. Properly weighed RVT-KIR mixture (1:1, 1:2, 1:3, 1:4) and RVT-KF90 mixture (1:1, 1:2, 1:3, 1:4) were taken into a beaker, and sufficient amount of methanol was added to dissolve the mixture. Subsequently, the mixture was stimulated for sufficient time to ensure a homogenous dispersion. In order to evaporate the solvent completely, the homogenously dispersed system was placed in a water-bath (placed in a closed environment) at 60$65^{\circ} \mathrm{C}$. The resultant dried residue was then kept in oven for 30 minutes at $50{ }^{\circ} \mathrm{C}$ to confirm complete removal of solvent and moisture. The moisture content of SD formulations was determined by moisture analyzer (Mettler Toledo, Switzerland) and was found less than $1 \%$ for all the formulations. Finally, the prepared SDs were persevered in a desiccator for 24 hours followed by named as S1, S2, S3, S4, S5, S6, S7 and S8. Before further analysis, SDs were crushed and sized uniformly using different sieve fractions and stored in desiccator (Table 1). ${ }^{38}$

Table 1. Rosuvastatin (RVT) formulations by physical mixing (PM) and solid dispersion (SD) techniques.

\begin{tabular}{ccccc}
\hline $\begin{array}{c}\text { Formulation } \\
\text { Codes }\end{array}$ & \multicolumn{3}{c}{ Ingredients } \\
\cline { 2 - 5 } & $\begin{array}{c}\text { Rosuvastatin Calcium } \\
\text { (RVT) }(\mathrm{mg})\end{array}$ & $\begin{array}{c}\text { Kollicoat }{ }^{\circledR} \text { IR (KIR) }(\mathrm{mg}) \\
\text { Kollidon® 90F (KF90) (mg) }\end{array}$ & Methanol \\
\hline F1 & 10 & 10 & - & Q.S. \\
F2 & 10 & 20 & - & Q.S. \\
F3 & 10 & 30 & - & Q.S. \\
F4 & 10 & 40 & - & Q.S. \\
F5 & 10 & - & 10 & Q.S. \\
F6 & 10 & - & 20 & Q.S. \\
F7 & 10 & - & 30 & Q.S. \\
F8 & 10 & - & 40 & Q.S. \\
S1 & 10 & 10 & - & Q.S. \\
S2 & 10 & 20 & - & Q.S. \\
S3 & 10 & 30 & - & Q.S. \\
S4 & 10 & 40 & - & Q.S. \\
S5 & 10 & - & 10 & Q.S. \\
S6 & 10 & - & 20 & Q.S. \\
S7 & 10 & - & 30 & Q.S. \\
S8 & 10 & - & 40 & Q.S. \\
\hline
\end{tabular}


Determination of the wavelength $\left(\lambda_{\max }\right)$. Firstly, $10 \mathrm{mg}$ of pure RVT was dissolved in $100 \mathrm{ml}$ of methanol, which was the primary stock solution $(100 \mu \mathrm{g} / \mathrm{ml})$. Then $10 \mu \mathrm{g} / \mathrm{ml}$ solution was prepared from the stock solution by adding distilled water. After preparing the stock solution, absorbance was measured by UV spectrophotometer (Shimadzu1700, Shimadzu Corp, Kyoto, Japan) at the range of 200- $400 \mathrm{~nm}$, and sharp peak was found at $242 \mathrm{~nm}^{39}$

Preparation of pH 6.8 buffer medium. Sodium dihydrogen phosphate (3.1202 g) and sodium phosphate dibasic dihydrate $(2.8392 \mathrm{~g})$ were weighed correctly and dissolved in $800 \mathrm{ml}$ distilled water.
Cyberscan $500 \mathrm{pH}$ meter, Singapore was used to check and adjust the $\mathrm{pH}$ at 6.8 using sodium hydroxide and $0.1 \mathrm{~N}$ hydrochloric acid. Then the amount of the medium was adjusted to $1000 \mathrm{ml}$ after obtaining the desired $\mathrm{pH}^{39}$

Calibration curve of RVT. A series of concentration of $0,4,8,12$ and $16 \mu \mathrm{g} / \mathrm{ml}$ of RVT was prepared through dilution with phosphate buffer of $\mathrm{pH} 6.8$ from the stock solution of pure RVT of concentration $100 \mu \mathrm{g} / \mathrm{ml}$. Then spectrophotometric analysis was performed by UV spectrophotometer at $242 \mathrm{~nm}$ (Figure 1).

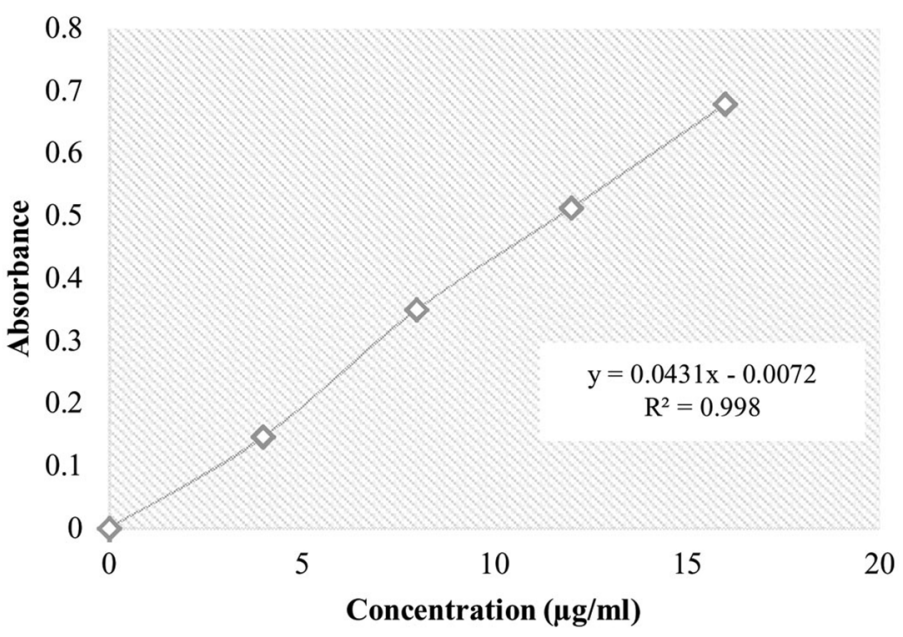

Figure 1. Calibration curve of RVT in phosphate buffer medium.

Dissolution studies of pure RVT, PMs and SDs. USP Type II dissolution apparatus (Erweka, Germany) stabled at $37 \pm 0.5^{\circ} \mathrm{C}$ and $50 \mathrm{rpm}$ was employed in this experiment to study the release kinetics of pure RVT, PMs and SDs system. ${ }^{18} 900 \mathrm{ml}$ phosphate buffer was used as dissolution medium and at every 10 minutes' interval, $5 \mathrm{ml}$ sample was withdrawn followed by filtration with Filter Paper No. 41 (Whatman plc, UK) and analyzed at $242 \mathrm{~nm}$ by UV-Visible spectrophotometer. To maintain the sink condition, dissolution medium was replenished simultaneously with $5 \mathrm{ml}$ of buffer media immediately after each sample withdrawal. ${ }^{18}$

Drug release kinetics and statistical analysis. Release of drug from its relevant dosage form is a major factor for evaluating its pharmacological effect. Therefore, estimation of drug release kinetics has an importance in pharmaceutical science. Different kinetics models such as zero order, first order, Higuchi, Hixson-Crowell and KorsmeyerPeppas models were used to know the RVT release pattern from PMs and SDs formulations. One-way analysis of variance (ANOVA) was also performed to dictate how drug release varies among these formulations. ${ }^{40}$

Fourier transform infrared spectroscopic (FTIR) analysis. RVT, KIR, KF90 and their optimized PMs and SDs formulations were scanned by FTIR (FTIR Spectrum Two TM L160000T of Perkin Elmer, USA) in a range of $4000-400 \mathrm{~cm}^{-1}$ with the scanning resolution of $1 \mathrm{~cm}^{-1}$. 
Thermal stability analysis by differential scanning calorimetry (DSC). DSC thermograms of RVT, KIR, KF90 and their optimized PMs and SDs formulations were obtained by DSC-60 thermal analyzer (Shimadzu corporation, Japan). 2.28-2.95 mg prepared samples were taken for the analysis and scanning was performed at a heating rate of $10^{\circ} \mathrm{C} / \mathrm{min}$ from $25^{\circ} \mathrm{C}-500^{\circ} \mathrm{C}$.

Surface morphology analysis by scanning electron microscope (SEM). Scanning Electron Microscope SEM-8100FM (Shimadzu, Japan) was used to analyze the surface of pure RVT, PMs and SDs by $2000 \mathrm{X}$ to $5500 \mathrm{X}$ magnification.

\section{Results and Discussion}

Drug release from PMs formulation of RVT. After performing dissolution studies, release rate of pure RVT was observed $63.1 \%$ in 60 minutes (Figure 2a). The formulations prepared by physical mixing with polymers KIR and KF90 showed an improved drug release rate than that of pure RVT. After 60 minutes, formulations F1, F2, F3 and F4 showed a drug release rate of $68.11 \%, 70.51 \%, 76.44 \%$ and $78.39 \%$ respectively (Figure 2a). Whether the formulations F5, F6, F7 and F8 showed a release rate of $71.38 \%, 74.44 \%, 82.39 \%$ and $84.14 \%$ respectively in 60 minutes (Figure 2a). These two polymers, KIR and KF90, which were used in the PMs are hydrophilic polymers. KIR has been applied in different SD formulations to boost the dissolution rate of indomethacin and also to increase the dissolution rate of clonazepam by solvent evaporation technique due to wetting ability. KF90 increases the dissolution rate of various poorly aqueous soluble drugs formulated using solid dispersion techniques such as lovastatin, itraconazole and repaglinide. ${ }^{41-45}$

\section{Drug release study from RVT SD} formulations prepared by solvent evaporation method. RVT solid dispersion (S1 to S8) formulations were prepared by solvent evaporation technique. Here also 1:1, 1:2, 1:3 and 1:4 ratios were considered for preparing SD formulations. In vitro dissolution study was accomplished for 60 minutes.
SD formulations, S1, S2, S3 and S4 exhibited cumulative drug release rate of $83.39 \%, 92.8 \%$, 98.23\% and $98.97 \%$ respectively whereas S5, S6, S7 and S8 formulations displayed cumulative drug release rate of $78.04 \%, 83.47 \%, 96.66 \%$ and $99.21 \%$ after 60 minutes. All the eight SD formulations showed much better release pattern than the pure RVT (Figure 2b).

Optimization of the formulations. Among the PM formulations made by KIR and KF90, F4 and F8 gave better cumulative percent drug release $(78.39 \%$ and $84.14 \%$ respectively) compared to their respective other PM formulations whereas S4 and S8 had given the best cumulative percent of drug release (98.97\% and $99.21 \%$ respectively) after 60 minutes among the respective SD formulations. Their cumulative percent of drug releases were compared to the pure RVT as well as PM formulations and found that S8 was the optimized formulation on the basis of cumulative percent drug release (Figure 2c). Though S8 was the best among all the formulations, however all other SD formulations maintained the USP mentioned drug release limit criteria after 60 minutes (Figure 2b).

RVT is crystalline in nature and there might be several reasons for better release rate in SD formulations with the water-soluble polymer KIR and KF90. This might be due to the reduction in crystallinity or its physical conversion into an amorphous shape. This might have somehow attributed to a reduction in particle size and an upsurge in the surface area thus resulting in a better dissolution rate. ${ }^{46}$

From cumulative percent of drug release pattern, among the PMs, F4 and F8 gave better drug release percent but S4 and S8 had given the best cumulative percent of drug release after 60 minutes (Figure 2c). Increasing the concentration of KIR and KF90 upturn the solubility of RVT both in PMs and SDs. From the comparison it also revealed that the release rate from different formulation prepared by different techniques increasing order would be SDs using KF90> SDs using KIR > PMs using KF90> PMs using KIR (Figure 2c). 

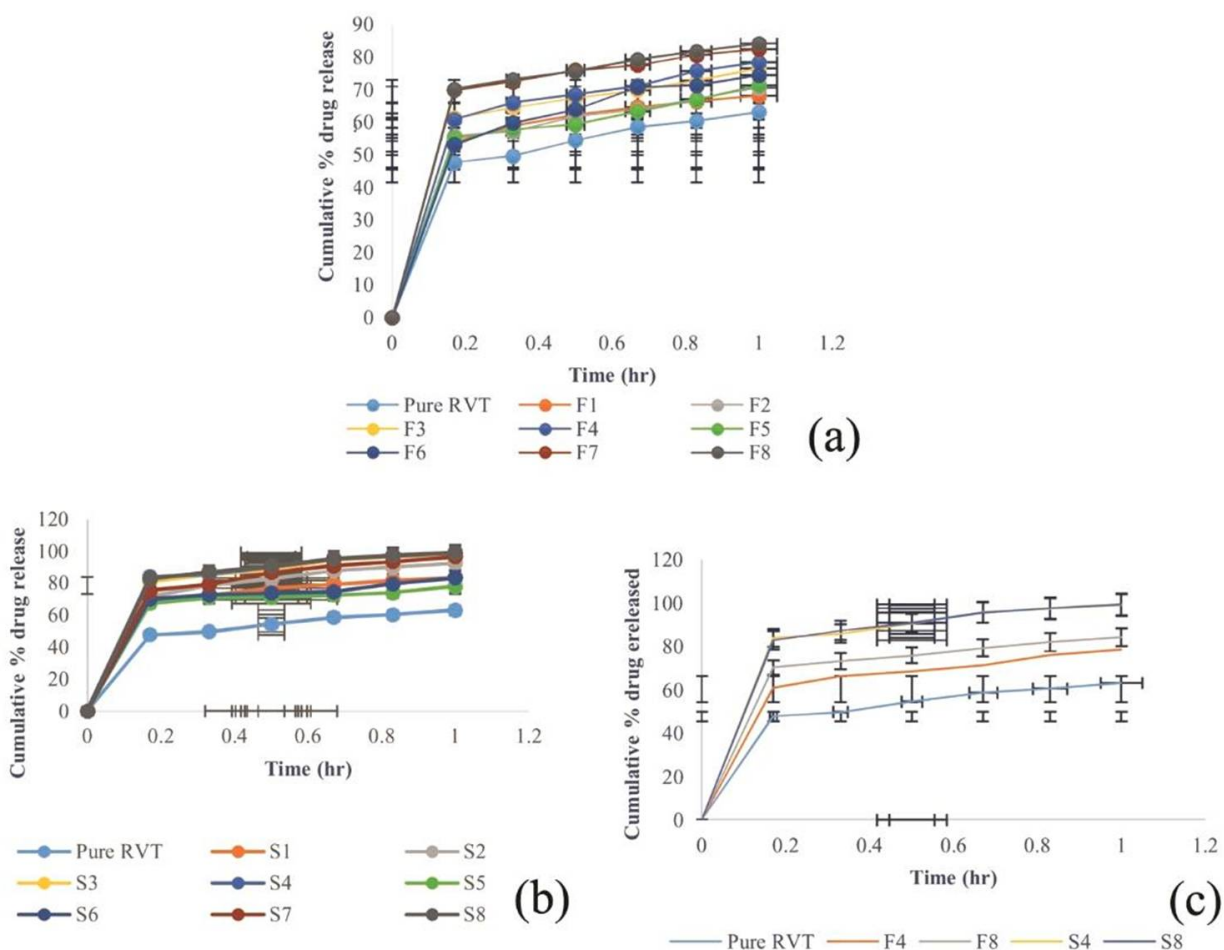

Figure 2. Comparison of cumulative percent drug release of pure RVT with (a) PM formulations (F1 to F8), (b) SD formulations (S1 to S8) prepared by solvent evaporation technique and (c) Comparison of formulations of SDs, PMs and pure RVT.

Drug release kinetic study and statistical analysis. Drug release kinetic study was achieved by different kinetic models like, zero order, first order, Higuchi, Hixson-Crowell and Korsmeyer-Peppas model. All the PM formulations were well fitted to the Higuchi model compared to other models and among the formulations F6 was the best fitted, where the $\mathrm{R}^{2}$ value was 0.8922 (Table 2). As Higuchi model was established for the study of water soluble and low soluble drugs in semisolid or solid matrices, it indicates that the formulations had developed solubility after physical mixing. Furthermore, SD formulations were fitted with the first order kinetics where S7 was the best fitted formulation with $\mathrm{R}^{2}$ value of 0.9473 (Table 2). However, according to the Korsmeyer-Peppas Model, all the formulations as well as the pure RVT followed the Super Case-II transport system as the value of $n$ was greater than 0.85 (Table 2).
To further inspect the effect of concentration of experimented polymers (KIR and KF90) on the dissolution profile of RVT, one-way ANOVA (analysis of variance) was performed to determine whether there were any statistically significant differences between the means of two groups. Results revealed that the $\mathrm{p}$-values were $<0.005$ for all the formulations which indicate the significant difference of the formulations. So, it supports that all the formulations showed the increased cumulative percent of drug release, when the concentrations of polymers were increased accordingly (Table 3 ).

Fourier transform infrared spectroscopy (FTIR). To assure compatibility of pure RVT with the experimented excipients, FTIR assays were conducted. Prominent peaks of the untreated active ingredient were found at 3417.86 (alcohol group), 2927.94 (O-H stretching carboxylic acid), 1550.77 (carbonyl group), 1151.50 (sulfone group) and 964.41 (aromatic C-H bending) $\mathrm{cm}^{-1}$ (Figure 3A). ${ }^{47,48}$ 
Additionally, in physical mixtures of KIR and KF90 with pure RVT, prominent peaks of RVT were found at almost the same position (KIR: 3429.43, 2926.01, $1548.84 \mathrm{~cm}^{-1}$; KF90: 3427.51, 2936.52, $1548.84 \mathrm{~cm}^{-}$ ${ }^{1}$ ) which ensure pure drug remained unchanged and no interaction with the polymers occurred in the physical mixture formulations (Figures 3D, 3E). The spectra of the solid dispersion systems, RVT-KIR, RVT-KF90 solid dispersion system also maintained consistency with the pure RVT spectrum (KIR: 3427.51, $2924.09 \mathrm{~cm}^{-1}$; KF90: 3429.33, 2926.01, $1548.91 \mathrm{~cm}^{-1}$; Figures 3F, 3G). The findings confirm that there was no significant modification took place while the pure drug was dispersed in the polymers utilizing solvent evaporation solid dispersion technique. As there was no stability issue, these polymers could be used readily to improve the dissolution profile RVT in large-scale production.

Table 2. Drug release kinetic of pure RVT, PMs and SDs formulations.

\begin{tabular}{|c|c|c|c|c|c|c|c|c|c|c|c|}
\hline \multirow[t]{2}{*}{$\begin{array}{l}\text { Formulation } \\
\text { Codes }\end{array}$} & \multirow[t]{2}{*}{$\begin{array}{l}\text { Drug: } \\
\text { Polymer }\end{array}$} & \multicolumn{2}{|c|}{ Zero Order } & \multicolumn{2}{|c|}{ First Order } & \multicolumn{2}{|c|}{ Higuchi Model } & \multicolumn{2}{|c|}{$\begin{array}{l}\text { Hixson- Crowell } \\
\text { Model }\end{array}$} & \multicolumn{2}{|c|}{$\begin{array}{c}\text { Korsmeyer-Peppas } \\
\text { Model }\end{array}$} \\
\hline & & $\mathrm{R}^{2}$ & $\mathrm{~K}_{0}$ & $\mathrm{R}^{2}$ & $\mathrm{~K}_{1}$ & $\mathrm{R}^{2}$ & $\mathrm{~K}_{\mathrm{h}}$ & $\mathrm{R}^{2}$ & $\mathrm{~K}_{\mathrm{HC}}$ & $\mathrm{R}^{2}$ & $\mathrm{n}$ \\
\hline $\mathrm{F} 1$ & $1: 1$ & 0.5694 & 50.502 & 0.6864 & -0.3928 & 0.8327 & 64.046 & 0.6448 & -1.1379 & 0.8608 & 1.0297 \\
\hline $\mathrm{F} 2$ & $1: 2$ & 0.5889 & 51.795 & 0.7229 & -0.4134 & 0.8404 & 64.908 & 0.6755 & -1.1858 & 0.8604 & 1.0309 \\
\hline F3 & $1: 3$ & 0.5652 & 55.286 & 0.7182 & -0.4845 & 0.8238 & 70.025 & 0.6628 & -1.3398 & 0.8567 & 1.0509 \\
\hline F4 & $1: 4$ & 0.5902 & 58.002 & 0.7630 & -0.5330 & 0.8439 & 72.757 & 0.7018 & -1.4476 & 0.8610 & 1.0591 \\
\hline F5 & $1: 1$ & 0.6011 & 52.127 & 0.7399 & -0.4185 & 0.8458 & 64.870 & 0.6915 & -1.1977 & 0.8614 & 1.0298 \\
\hline F6 & $1: 2$ & 0.6542 & 58.149 & 0.8123 & -0.5030 & 0.8922 & 71.241 & 0.7594 & -1.4007 & 0.8762 & 1.0519 \\
\hline F7 & $1: 3$ & 0.5236 & 58.814 & 0.6936 & -0.5873 & 0.7928 & 75.922 & 0.6291 & -1.5399 & 0.8490 & 1.0728 \\
\hline F8 & $1: 4$ & 0.5396 & 60.534 & 0.7321 & -0.6312 & 0.8049 & 77.561 & 0.6601 & -1.6268 & 0.8513 & 1.0771 \\
\hline S1 & $1: 1$ & 0.4878 & 53.526 & 0.6026 & -0.4726 & 0.7613 & 70.150 & 0.5586 & -1.3005 & 0.8519 & 1.0773 \\
\hline S2 & $1: 2$ & 0.5218 & 58.227 & 0.6918 & -0.5808 & 0.7861 & 74.973 & 0.6281 & -1.5222 & 0.8620 & 1.1045 \\
\hline S3 & $1: 3$ & 0.5962 & 72.519 & 0.9346 & -1.2753 & 0.8499 & 90.832 & 0.8345 & -2.6149 & 0.8535 & 1.1181 \\
\hline S4 & $1: 4$ & 0.5347 & 71.588 & 0.9374 & -1.7046 & 0.8922 & 71.241 & 0.8179 & -2.9767 & 0.8518 & 1.1217 \\
\hline S5 & $1: 1$ & 0.5389 & 60.465 & 0.7253 & -0.6254 & 0.8059 & 77.570 & 0.6556 & -1.6177 & 0.8430 & 1.0554 \\
\hline S6 & $1: 2$ & 0.5937 & 69.724 & 0.8859 & -0.9850 & 0.8498 & 87.510 & 0.7876 & -2.2363 & 0.8469 & 1.0692 \\
\hline S7 & $1: 3$ & 0.5532 & 72.120 & 0.9413 & -1.5525 & 0.8175 & 91.970 & 0.8224 & -2.8724 & 0.8611 & 1.1131 \\
\hline S8 & $1: 4$ & 0.5502 & 73.265 & 0.9034 & -2.0066 & 0.8130 & 93.434 & 0.9046 & -3.8799 & 0.8510 & 1.1202 \\
\hline RVT & - & 0.6309 & 48.063 & 0.7462 & -0.3493 & 0.8725 & 59.295 & 0.7065 & -1.0384 & 0.8704 & 1.0072 \\
\hline
\end{tabular}

Differential scanning calorimetry (DSC) analysis. DSC study demonstrated that the melting point of the pure RVT, KIR, and KF90 was in $86.38^{\circ} \mathrm{C}, \quad 115.49^{\circ} \mathrm{C}$, and $154.13^{\circ} \mathrm{C}$ respectively (Figure 4A, 4B, 4C). Increase in melting point was observed for RVT-KIR PM formulations whereas the RVT-KF90 PM formulations remained stable up to $152.03^{\circ} \mathrm{C}$ (Figure 4D, 4E). DSC thermogram of PM formulations displayed different sharp endothermic peaks from the pure RVT which is due to the crystallinity form change of this drug. ${ }^{47}$ In case of SD formulations, the melting point was in between pure RVT and KIR for RVT-KIR SD formulations, and a decrease in melting point was observed for the RVTKF90 SD formulations (Figure 4F, 4G). The peaks of the pure RVT were disappeared in the DSC curve of SD formulations due to the conversion of RVT crystal structure to the amorphous state. ${ }^{47}$

Scanning electron microscopic (SEM) analysis. Surface morphology of the pure RVT, PMs and SDs have been inspected by performing SEM analysis. The micrograph provides a detailed illustration of drug particle state before and after the treatment with excipients. Untreated pure RVT crystal appeared to be with rough surfaces where crystals of PM of KIR with RVT displayed shiny 
surfaces (Figure 5A, 5B). Reduced crystallinity was observed for the PM of KF90 (Figure 5C). The feature explains better dissolution properties of the F5, F6, F7, and F8 formulation corresponding to the F1, F2, F3, and F4 formulation respectively. Furthermore, in SD of KIR with RVT, the pure drug particles were found to be dispersed in the excipient (Figure 5D). On the other hand, SD of KF90 with RVT was in amorphous state (Figure 5E). Herein, the excipient and the pure drug content formed a homogenous dispersion system and obtained a better dissolution profile than other formulations.

Table 3. ANOVA analysis of PMs and SDs (solvent evaporation method) formulations of RVT with two polymers in different ratios.

\begin{tabular}{|c|c|c|c|c|c|c|c|c|}
\hline $\begin{array}{l}\text { Formulation } \\
\text { Codes }\end{array}$ & $\begin{array}{l}\text { Drug: } \\
\text { Polymer }\end{array}$ & Source of Variation & SS & Df & Ms & $\mathrm{F}$ & P-value & $\mathrm{F}_{\text {crit }}$ \\
\hline \multirow[t]{2}{*}{$\mathrm{F} 1$} & $1: 1$ & Between Groups & 1923.252 & 1 & 1923.252 & 3.680 & 0.079 & 4.747 \\
\hline & & Within Groups & 6271.361 & 12 & 522.613 & & & \\
\hline \multirow[t]{2}{*}{$\mathrm{F} 2$} & $1: 2$ & Between Groups & 1973.744 & 1 & 1973.744 & 3.740 & 0.077 & 4.747 \\
\hline & & Within Groups & 6332.943 & 12 & 527.745 & & & \\
\hline \multirow[t]{2}{*}{$\mathrm{F} 3$} & $1: 3$ & Between Groups & 2917.458 & 1 & 2917.458 & 5.005 & 0.045 & 4.747 \\
\hline & & Within Groups & 6994.697 & 12 & 582.891 & & & \\
\hline \multirow[t]{2}{*}{$\mathrm{F} 4$} & $1: 4$ & Between Groups & 3171.938 & 1 & 3171.938 & 5.271 & 0.040 & 4.747 \\
\hline & & Within Groups & 7220.782 & 12 & 601.732 & & & \\
\hline \multirow[t]{2}{*}{ F5 } & $1: 1$ & Between Groups & 1911.082 & 1 & 1911.082 & 3.637 & 0.081 & 4.747 \\
\hline & & Within Groups & 6306.207 & 12 & 525.517 & & & \\
\hline \multirow[t]{2}{*}{ F6 } & $1: 2$ & Between Groups & 2392.333 & 1 & 2392.333 & 4.216 & 0.062 & 4.747 \\
\hline & & Within Groups & 6809.059 & 12 & 567.422 & & & \\
\hline \multirow[t]{2}{*}{ F7 } & $1: 3$ & Between Groups & 4418.688 & 1 & 4418.688 & 6.692 & 0.024 & 4.747 \\
\hline & & Within Groups & 7923.964 & 12 & 660.330 & & & \\
\hline \multirow[t]{2}{*}{ F8 } & $1: 4$ & Between Groups & 4606.109 & 1 & 4606.109 & 6.852 & 0.022 & 4.747 \\
\hline & & Within Groups & 8067.088 & 12 & 672.257 & & & \\
\hline \multirow[t]{2}{*}{$\mathrm{S} 1$} & $1: 1$ & Between Groups & 4594.145 & 1 & 4594.145 & 6.838 & 0.023 & 4.747 \\
\hline & & Within Groups & 8061.748 & 12 & 671.812 & & & \\
\hline \multirow[t]{2}{*}{ S2 } & $1: 2$ & Between Groups & 6155.114 & 1 & 6155.114 & 8.071 & 0.015 & 4.747 \\
\hline & & Within Groups & 9150.976 & 12 & 762.581 & & & \\
\hline \multirow[t]{2}{*}{ S3 } & $1: 3$ & Between Groups & 7981.174 & 1 & 7981.174 & 9.490 & 0.010 & 4.747 \\
\hline & & Within Groups & 10091.81 & 12 & 840.9839 & & & \\
\hline \multirow[t]{2}{*}{ S4 } & $1: 4$ & Between Groups & 8488.484 & 1 & 8488.484 & 9.826 & 0.008 & 4.747 \\
\hline & & Within Groups & 10366.9 & 12 & 863.908 & & & \\
\hline \multirow[t]{2}{*}{ S5 } & $1: 1$ & Between Groups & 3594.888 & 1 & 3594.888 & 5.865 & 0.032 & 4.747 \\
\hline & & Within Groups & 7355.074 & 12 & 612.923 & & & \\
\hline \multirow[t]{2}{*}{ S6 } & $1: 2$ & Between Groups & 4274.56 & 1 & 4274.560 & 6.543 & 0.025 & 4.747 \\
\hline & & Within Groups & 7839.36 & 12 & 653.280 & & & \\
\hline \multirow[t]{2}{*}{ S7 } & $1: 3$ & Between Groups & 6972.322 & 1 & 6972.322 & 8.678 & 0.012 & 4.747 \\
\hline & & Within Groups & 9641.446 & 12 & 803.454 & & & \\
\hline \multirow[t]{2}{*}{ S8 } & $1: 4$ & Between Groups & 8388.317 & 1 & 8388.317 & 9.794 & 0.009 & 4.747 \\
\hline & & Within Groups & 10277.3 & 12 & 856.442 & & & \\
\hline
\end{tabular}



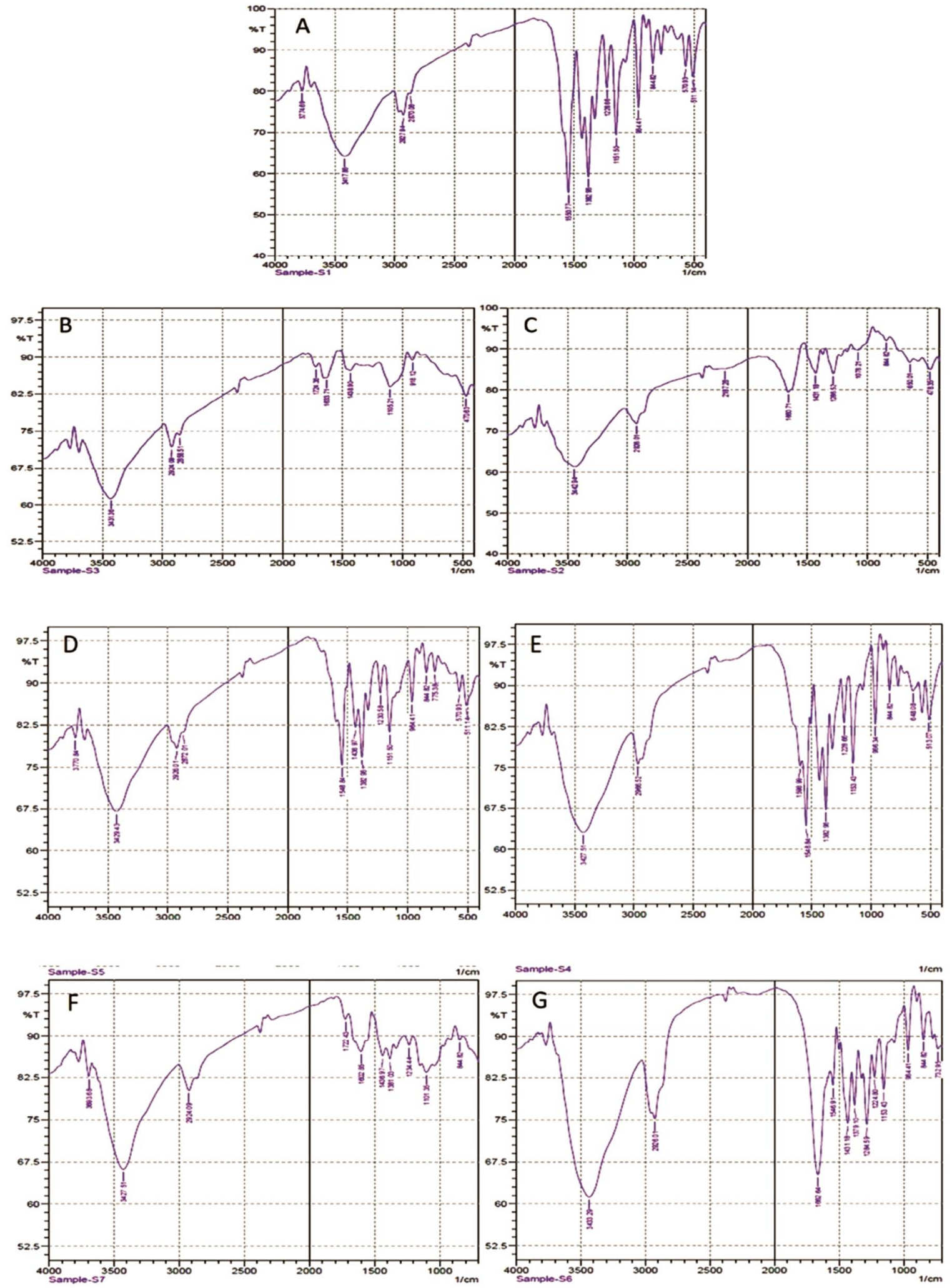

Figure 3. FTIR spectrum of (A) RVT, (B) KIR, (C) KF90 (D) PM of RVT with KIR, (E) PM of RVT with KF90, (F) SD of RVT with KIR, (G) SD of RVT with KF90. 

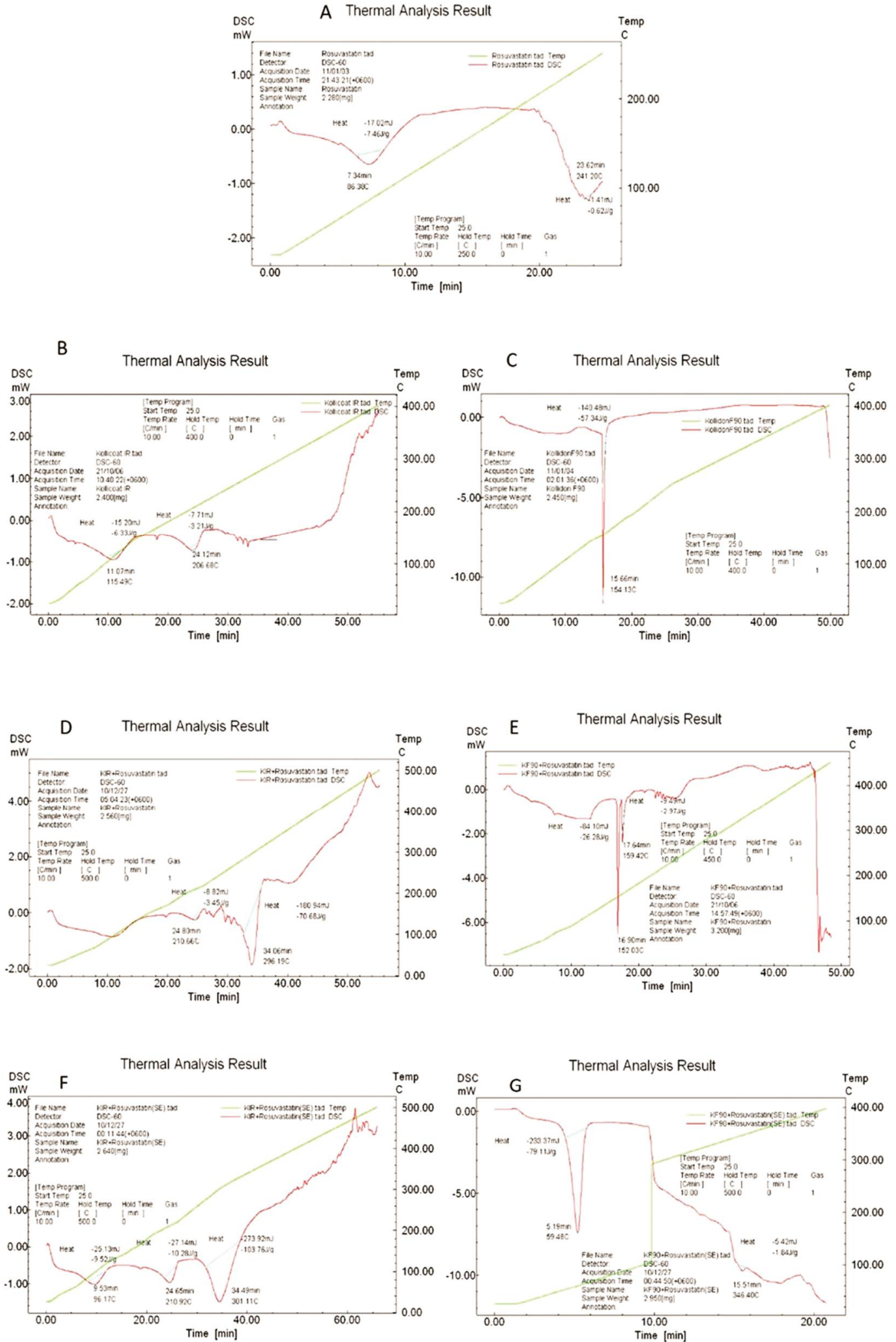

Figure 4. DSC thermogram of (A) RVT, (B) KIR, (C) KF90 (D) PM of RVT with KIR, (E) PM of RVT with KF90, (F) SD of RVT with KIR and (G) SD of RVT with KF90. 


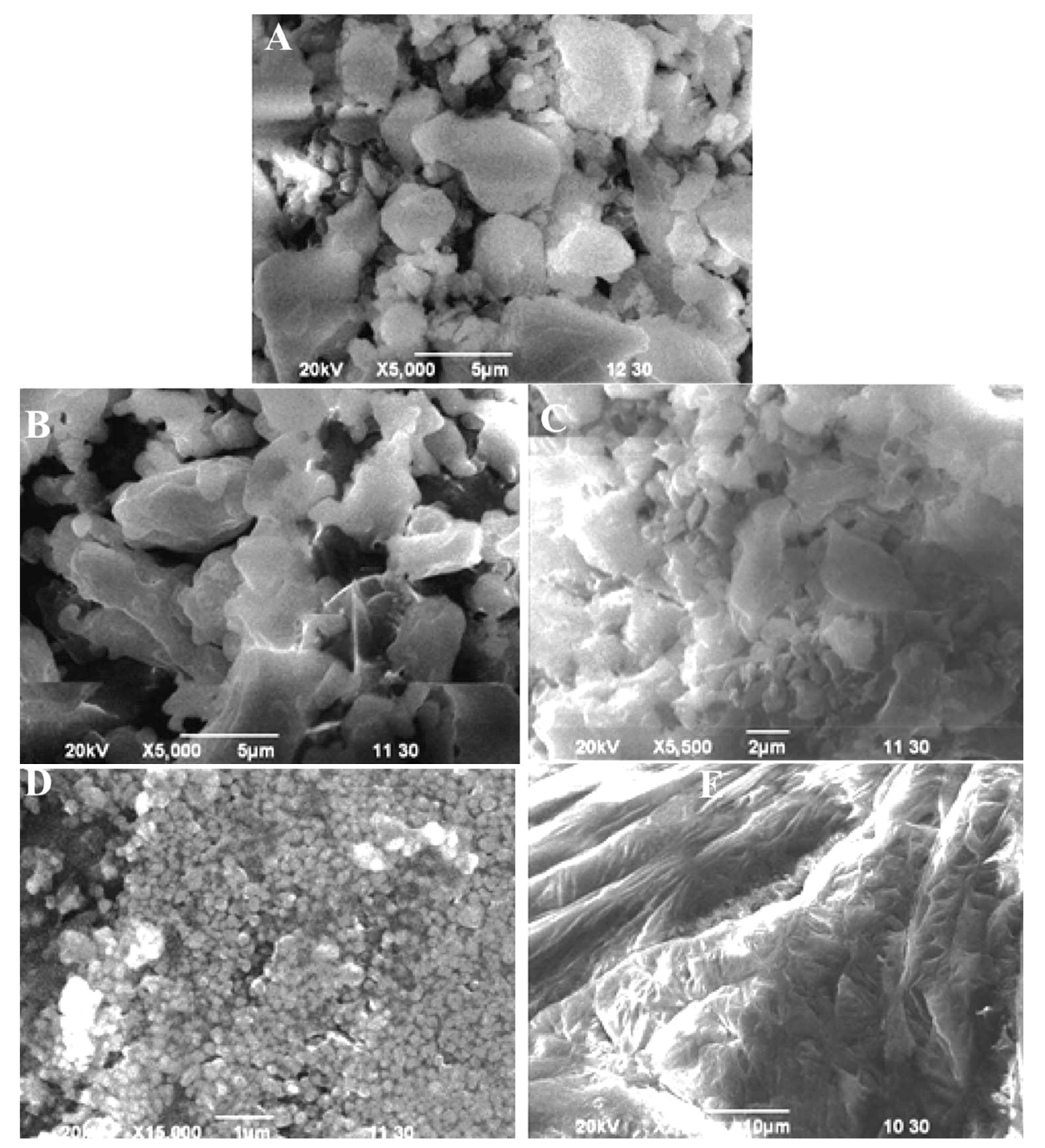

Figure 5. SEM of (A) RVT at 5000 magnifications, (B) PM of RVT with KIR at 5000 magnifications, (C) PM of RVT with KF90 at 5500 magnifications, (D) SD of RVT with KIR at 15000 magnifications and (E) SD of RVT with KF90 at 2000 magnifications.

\section{CONCLUSION}

Current study investigated that how the dissolution profile of sparingly soluble RVT can be improved by KIR and KF90 using a solid dispersion (SD) approach (solvent evaporation technique). Among the prepared formulations, S8 had given the best cumulative percent drug release after 60 minutes. This revealed that increasing concentration of KIR and KF90 improved the dissolution of RVT both in PMs and SDs. From the comparison, it is also demonstrated that the release rate from different prepared formulations, increasing order would be SDs using KF90> SDs using KIR> PMs using
KF90> PMs using KIR. The physicochemical stability and amorphous characteristics of these formulations were also supported by FTIR, DSC and SEM data. No significant peak deviations were observed in FTIR results for PMs and SDs of KIR with RVT in comparison to the pure RVT spectrum. Reduced crystallinity of RVT was also obtained in PM formulations and SD formulations through SEM analysis, where it was illustrated that the RVT in KF90 dispersion system almost transformed into amorphous state which was responsible for better dissolution profile. The study suggested that KF90 is a potential drug carrier for RVT SD system to 
improve the dissolution of pure RVT, and the optimized ratio for the RVT to KF90 was found 1:4. However, further study is needed for ensuring more precise SD formulations and in vivo assays for manufacturing SD RVT tablet dosage form.

\section{ACKNOWLEDGEMENTS}

The authors acknowledge the Centre for Advanced Research in Sciences (CARS), University of Dhaka, Bangladesh and Bangladesh Reference Institute for Chemical Measurement (BRiCM), Dhaka, Bangladesh for giving the opportunity to use their laboratory facilities. This research was funded by the Ministry of Science and Technology (MoST), Government Republic of Bangladesh as a Research and Development (R\&D) project.

\section{REFERENCES}

1. Naqvi, A., Ahmad, M., Minhas, M. U., Khan, K. U., Batool, F., and Rizwan, A. 2020. Preparation and evaluation of pharmaceutical co-crystals for solubility enhancement of atorvastatin calcium. Polym. Bull. 77, 6191-6211.

2. Amidon, G. L., Lennernäs, H., Shah, V. P. and Crison, J. R. A 1995. Theoretical basis for a biopharmaceutic drug classification: the correlation of in vitro drug product dissolution and in vivo bioavailability. Pharm. Res. An Off. J. Am. Assoc. Pharm. Sci. 12, 413-420.

3. Godase, C. B., Babar, A. L. and Gopal, A. B. 2020. Concise review on methods of solubility enhancement. Int. Pharm. Sci. 11, 109.

4. Gao, Y., Glennon, B., He, Y. and Donnellan, P. 2021. Dissolution kinetics of a bcs class ii active pharmaceutical ingredient: diffusion-based model validation and prediction. ACS Omega 6, 8056-8067.

5. Noyes, A. A. and Whitney, W. R. 1897. The rate of solution of solid substances in their own solutions. J. Am. Chem. Soc. 19, 930-934.

6. Nernst, W. 2017. Theorie der Reaktionsgeschwindigkeit in heterogenen Systemen. Zeitschrift für Phys. Chemie 47U, 52-55.

7. Leuner, C. and Dressman, J. 2000. Improving drug solubility for oral delivery using solid dispersions. E. J. Pharm. Biopharm. 50, 47-60.

8. Jatwani, S., Rana, A. C., Singh, G. and Aggarwal, G. 2012. An overview on solubility enhancement techniques for poorly soluble drugs and solid dispersion as an eminent strategic approach. Int. J. Pharm. Sci. Res. 3, 942.

9. Sekiguchi, K. and Obi, N. 1961. Studies on absorption of eutectic mixture. i. a comparison of the behavior of eutectic mixture of sulfathiazole and that of ordinary sulfathiazole in man. Chem. Pharm. Bull. 9, 866-872.
10. Felton, L. A. 2013. Remington's Essentials of Pharmaceutics. Pharmaceutical Press.

11. Alshehri, S., Imam, S.S., Hussain, A., Altamimi, M.A., Alruwaili, N.K., Alotaibi, F., Alanazi, A. and Shakeel, F. 2020. Potential of solid dispersions to enhance solubility, bioavailability, and therapeutic efficacy of poorly watersoluble drugs: newer formulation techniques, current marketed scenario and patents. Drug Delivery 27, 1625-1643.

12. Serajuddln, A. T. M. 1999. Solid dispersion of poorly watersoluble drugs: early promises, subsequent problems, and recent breakthroughs. J. Pharm. Sci. 88, 1058-1066.

13. Zhang, X., Xing, H., Zhao, Y. and Ma, Z. 2018. Pharmaceutical dispersion techniques for dissolution and bioavailability enhancement of poorly water-soluble drugs. Pharmaceutics 10, 74.

14. Belozerova, N.M., Bilski, P., Jarek, M., Jenczyk, J., Kichanov, S.E., Kozlenko, D.P., Mielcarek, J., Pajzderska, A. and Wąsicki, J. 2020. Exploring the molecular reorientations in amorphous rosuvastatin calcium. RSC Adv. 10, 3358533594.

15. Quirk, J., Thornton, M. and Kirkpatrick, P. 2003. Rosuvastatin calcium. Market analysis. Nat. Rev. Drug Discov. 2, 769-770.

16. Luvai, A., Mbagaya, W., Hall, A. S. Barth, J. H. 2012. Rosuvastatin: a review of the pharmacology and clinical effectiveness in cardiovascular disease. Clin. Med. Insights Cardiol. 6, 17-33.

17. Zhang, L., Zhang, S., Yu, Y., Jiang, H. and Ge, J., 2020. Efficacy and safety of rosuvastatin vs. atorvastatin in lowering LDL cholesterol : a meta-analysis of trials with East Asian populations. Herz 45, 594-602.

18. Al-Heibshy, F.N., Başaran, E., Arslan, R., Öztürk, N., Erol, K. and Demirel, M., 2020. Physicochemical characterization and pharmacokinetic evaluation of rosuvastatin calcium incorporated solid lipid nanoparticles. Int. J. Pharm. 578, 119106.

19. Alshora, D.H., Ibrahim, M.A., Elzayat, E., Almeanazel, O.T. and Alanazi, F. 2018. Rosuvastatin calcium nanoparticles: improving bioavailability by formulation and stabilization codesign. PLoS One, 13, p.e0200218.

20. Rizwanullah, M., Amin, S. and Ahmad, J. 2016. Improved pharmacokinetics and antihyperlipidemic efficacy of rosuvastatin-loaded nanostructured lipid carriers. J. Drug Targ. 25, 58-74.

21. Balakumar, K., Raghavan, C.V. and Abdu, S. 2013. Self nanoemulsifying drug delivery system (SNEDDS) of rosuvastatin calcium: design, formulation, bioavailability and pharmacokinetic evaluation. Colloids Surfaces B Biointerfaces 112, 337-343.

22. Kamel, A. O. and Mahmoud, A. A. 2013. Enhancement of human oral bioavailability and in vitro antitumor activity of rosuvastatin via spray dried self-nanoemulsifying drug delivery system. J. Biomed. Nanotechnol. 9, 26-39. 
23. Karasulu, H.Y., Gundogdu, E., Turk, U.O., Turgay, T., Apaydin, S., Simsir, I.Y., Yilmaz, C. and Karasulu, E. 2018. Enhancing solubility and bioavailability of rosuvastatin into self nanoemulsifying drug delivery system. Curr. Drug Deliv. 15, 1072-1082.

24. Al-Shdefat, R., Anwer, M.K., Fayed, M.H., Alsulays, B.B., Tawfeek, H.M., Abdel-Rahman, R.F. and Soliman, G.A. 2020. Preparation and evaluation of spray dried rosuvastatin calcium-PVP microparticles for the improvement of serum lipid profile. J. Drug Deliv. Sci. Technol. 55, 101342.

25. Sarfraz, R. M., Ahmad, M., Mahmood, A., Akram, M. R and Abrar, A. 2017. Development of $\beta$-cyclodextrin-based hydrogel microparticles for solubility enhancement of rosuvastatin: an in vitro and in vivo evaluation. Drug Des. Devel. Ther. 11, 3083.

26. Sarfraz, R. M., Ahmad, M., Mahmood, A., Minhas, M. U. and Yaqoob, A. 2017. Development and evaluation of rosuvastatin calcium based microparticles for solubility enhancement: an in vitro study. Adv. Polym. Technol. 36, 433-441.

27. Sarfraz, R. M., Ahmad, M., Mahmood, A. and Ijaz, H. 2018. Development, in vitro and in vivo evaluation of $\mathrm{ph}$ responsive $\beta$-cd-comethacrylic acid-crosslinked polymeric microparticulate system for solubility enhancement of rosuvastatin calcium. Polym. - Plast. Technol. Eng. 57, 11751187.

28. Kamble, P. R., Shaikh, K. S. and Chaudhari, P. D. 2014. Application of liquisolid technology for enhancing solubility and dissolution of rosuvastatin. Adv. Pharm. Bull. 4, 197204.

29. Kapure, V. J., Pande, V. V. and Deshmukh, P.K. 2013. Dissolution Enhancement of Rosuvastatin Calcium by Liquisolid Compact Technique. J. Pharm. 2013, 1-9.

30. Swathi, T., Krishna, M. V., Kumar, D. S. and Krishnaveni, J. 2013. Enhancement of solubility and dissolution rate of rosuvastatin by using solid dispersion technique. J. Pharm. Sci. Innov. 2, 36-40.

31. Fouad, E. A., El-Badry, M., Neau, S. H., Alanazi, F. K. and Alsarra, I. A. 2011. Technology evaluation: Kollicoat IR. Expert Opin. Drug Deliv. 8, 693-703.

32. Janssens, S., de Armas, H. N., Remon, J. P. and Van den Mooter, G. 2007. The use of a new hydrophilic polymer, Kollicoat IR®, in the formulation of solid dispersions of itraconazole. Eur. J. Pharm. Sci. 30, 288-294.

33. Runa, F., Sarkar, M. R., Sultana, R., Jahan, K. and Labu, Z. K. 2013. Study on dissolution improvement of allopurinol by co-grinding and fusion method using solid dispersion technique. J. Biomed. Pharm. Res. 2, 1-7.

34. Kadir, M.F., Alam, M.R., Rahman, A.B., Jhanker, Y.M., Shams, T. and Khan, R.I., 2012. Study of binary and ternary solid dispersion of spironolactone prepared by coprecipitation method for the enhancement of oral bioavailability. J. Appl. Pharm. Sci. 2, 117-122.
35. Albarahmieh, E., Albarahmieh, M. and Alkhalidi, B. A. 2018. Fabrication of hierarchical polymeric thin films by spin coating toward production of amorphous solid dispersion for buccal drug delivery system: preparation, characterization, and in vitro release investigations. J. Pharm. Sci. 107, 31123122.

36. Xu, L., Li, S.M., Wang, Y., Wei, M., Yao, H.M. and Sunada, H., 2009. Improvement of dissolution rate of ibuprofen by solid dispersion systems with Kollicoat IR using a pulse combustion dryer system. J. Drug Deliv. Sci. Technol. 19, 113-118.

37. Dreu, R., Ilić, I. and Srčič, S. 2011. Development of a multiple-unit tablet containing enteric-coated pellets. Pharmaceut. Dev. Technol. 16, 118-126.

38. Nicolescu, C., Aramă, C., Nedelcu, A., Monciu, C. M. 2010. Phase solubility studies of the inclusion complexes of repaglinide with $\beta$-cyclodextrin and $\beta$-cyclodextrin derivatives. Farmacia 58, 620-628.

39. Messa, R. and Ampati, S. 2016. Comparative studies for enhancement of the dissolution profile of pitavastatin. Int. J. Med. Nanotechnol. 3, 311-315.

40. Barzegar-Jalali, M., 2008. Kinetic analysis of drug release from nanoparticles. J. Pharm. Pharm. Sci. 11, 167-177.

41. Shirke, S. H., Shete, A. S. and Doijad, R. C. 2015. Enhancement of dissolution rate of indomethacin by kollicoat IR based solid dispersions. Der Pharm. Lett. 7, 64-73.

42. Minhaz, M. A., Rahman, M. M., Ahsan, M. Q., Khalipha, A. B. R. and Chowdhury, M. R. 2012. Dissolution enhancement of poorly soluble drug by solvent evaporation method using hydrophilic polymer: a solid dispersion technique. Int. J. Pharm. Life Sci. 1.

43. Sarkar, M.R., Sikdar, K.Y.K., Monjur-Al-Hossain, A.S.M. and Sharmin, N., 2021. Improving the drug dissolution profile of poorly aqueous soluble lovastatin using hydrophilic polymers by solid dispersion and physical mixing techniques. Natl. J. Physiol. Pharm. Pharmacol. 11, 1.

44. Chowdary, K.P.R. and Rao, S.S., 2000. Investigation of dissolution enhancement of itraconazole by solid dispersion in superdisintegrants. Drug Dev. Ind. Pharm. 26, 1207-1211.

45. Sarkar, R., Sultana, R. and Faroque, A. B. M. 2018. Improvement of solubility of repaglinide by physical mixing and melt solvent method. Saudi J. Med. Pharm. Sci. 4, 199206.

46. Tous, S. S., El Sayed, A. M., Abd El Mohsen, M. G., Agban, M. N. andBoushra, M. F. 2012. Enhancement of nalidixic acid solubility via cosolvency and solid dispersion. J. Drug Deliv. Sci. Technol. 22, 341-346.

47. Verma, R., Kaushik, A., Almeer, R., Rahman, M.H., AbdelDaim, M.M. and Kaushik, D. 2021. Improved Pharmacodynamic Potential of Rosuvastatin by SelfNanoemulsifying Drug Delivery System: An in vitro and in vivo Evaluation. Int. J. Nanomed. 16, 905.

48. Mubtasim, N., Kabir, E.R., Podder, A.K. and Bhadra, S. 2016. A pragmatic approach to the analysis of a combination formulation. Saudi Pharmaceut. J. 24, 689-697. 